The Journal of Symbolic Logid

Volume 00, Number 0, XXX 0000

\title{
DOMINIONS AND PRIMITIVE POSITIVE FUNCTIONS
}

\author{
MIGUEL CAMPERCHOLI
}

\begin{abstract}
Let $\mathbf{A} \leq \mathbf{B}$ be structures, and $\mathcal{K}$ a class of structures. An element $b \in B$ is dominated by $\mathbf{A}$ relative to $\mathcal{K}$ if for all $\mathbf{C} \in \mathcal{K}$ and all homomorphisms $g, g^{\prime}: \mathbf{B} \rightarrow \mathbf{C}$ such that $g$ and $g^{\prime}$ agree on $A$, we have $g b=g^{\prime} b$. Our main theorem states that if $\mathcal{K}$ is closed under ultraproducts, then $\mathbf{A}$ dominates $b$ relative to $\mathcal{K}$ if and only if there is a partial function $F$ definable by a primitive positive formula in $\mathcal{K}$ such that $F^{\mathbf{B}}\left(a_{1}, \ldots, a_{n}\right)=b$ for some $a_{1}, \ldots, a_{n} \in A$. Applying this result we show that a quasivariety of algebras $\mathcal{Q}$ with an $n$-ary near-unanimity term has surjective epimorphisms if and only if $\mathbb{S P}_{n} \mathbb{P}_{u}\left(\mathcal{Q}_{\mathrm{RSI}}\right)$ has surjective epimorphisms. It follows that if $\mathcal{F}$ is a finite set of finite algebras with a common near-unanimity term, then it is decidable whether the (quasi)variety generated by $\mathcal{F}$ has surjective epimorphisms.
\end{abstract}

§1. Introduction. Let be $\mathcal{L}$ a first order language. Given $\mathcal{L}$-structures $\mathbf{A} \leq$ $\mathbf{B}$ and $\mathcal{K}$ a class of $\mathcal{L}$-structures, an element $b \in B$ is dominated [13] by $\mathbf{A}$ relative to $\mathcal{K}$ provided that for every $\mathbf{C} \in \mathcal{K}$ and all homomorphisms $g, g^{\prime}: \mathbf{B} \rightarrow \mathbf{C}$ such that $\left.g\right|_{A}=\left.g^{\prime}\right|_{A}$, we have $g b=g^{\prime} b$. That is, if $g$ and $g^{\prime}$ agree on $A$, then they must agree on $b$. The dominion of $\mathbf{A}$ in $\mathbf{B}$ relative to $\mathcal{K}$, denoted by $\operatorname{dom}_{\mathbf{B}}^{\mathcal{K}} \mathbf{A}$, is the set of all elements in $B$ that are dominated by $\mathbf{A}$. At first glance the definition may suggest that $A$ generates $\operatorname{dom}_{\mathbf{B}}^{\mathcal{K}} \mathbf{A}$, but on closer inspection this does not make sense. As $\mathbf{A}$ is a substructure of $\mathbf{B}$, generating with $A$ will yield exactly A. However, as the main result of this article shows, the intuition that $A$ acts as a set of generators of $\operatorname{dom}_{\mathbf{B}}^{\mathcal{K}} \mathbf{A}$ is not far off. In fact, if $\mathcal{K}$ is closed under ultraproducts, we prove that $A$ actually "generates" $\operatorname{dom}_{\mathbf{B}}^{\mathcal{K}} \mathbf{A}$, only that the generation is not through the fundamental operations but rather through primitive positive definable partial functions. Let us take a look at an example. Write $\mathcal{D}_{01}$ for the class of bounded distributive lattices, let $\mathbf{B}:=\mathbf{2} \times \mathbf{2}$, and let $\mathbf{A}$ be the sublattice of $\mathbf{B}$ with universe $\{\langle 0,0\rangle,\langle 0,1\rangle,\langle 1,1\rangle\}$. As 01-lattice homomorphisms map pairs of complemented elements to pairs of complemented elements, and every element in a distributive lattice has at most one complement, it follows that $\langle 1,0\rangle \in \operatorname{dom}_{\mathbf{B}}^{\mathcal{K}} \mathbf{A}$. The key element to take away from this argument is that $\langle 1,0\rangle$ is generated by $A$ if we add the complementation operation to $\mathbf{B}$.

2000 Mathematics Subject Classification. 18A20; 03C05, 03C60, 03C40, 08C15.

Key words and phrases. Epimorphism, dominion, epic substructure, Beth definability, definable function, Isbell's Zigzag Theorem.

The author was partially supported by Conicet, Secyt-UNC project 30720150100529CB, and ANPCyT-PICT-2013-2011.

(C) 0000 , Association for Symbolic Logic $0022-4812 / 00 / 0000-0000 / \$ 00.00$ 
Since this (partial) operation is defined in every member of $\mathcal{D}_{01}$ by the existential positive formula

$$
\varphi(x, y):=x \wedge y=0 \& x \vee y=1,
$$

it is preserved by all relevant maps. This situation can be generalized as follows. Suppose $\mathcal{K}$ is any class, and $\varphi(\bar{x}, y)$ is an existential positive formula such that for every $\mathbf{C} \in \mathcal{K}$ and all $c_{1}, \ldots, c_{n} \in C$ there is at most one $d \in C$ such that $\varphi(\bar{c}, d)$ holds in $\mathbf{C}$. Now if $\mathbf{A} \leq \mathbf{B}$ and $b \in B$ is such that $\mathbf{B} \vDash \varphi(\bar{a}, b)$ for some elements $\bar{a}$ from $A$, then $b \in \operatorname{dom}_{\mathbf{B}}^{\mathcal{K}} \mathbf{A}$. Theorem 3 below says that for $\mathcal{K}$ closed under ultraproducts the converse is also true; that is, dominions relative to $\mathcal{K}$ are generated by existential positive definable partial operations.

Since their introduction in [13], there has been vigorous work on dominions; a well written survey on the subject is [8]. Results on dominions range from characterizations for specific classes of structures (e.g., semigroups [13, 11], lattices [19], etc.), to more general results such as [3], where dominions are characterized in quasivarieties of algebras in terms of amalgamated free products.

The notion of dominion was introduced by Isbell to study epimorphisms. Recall that a homomorphism $h: \mathbf{A} \rightarrow \mathbf{B}$ is a $\mathcal{K}$-epimorphism if for every $\mathbf{C} \in \mathcal{K}$ and homomorphisms $g, g^{\prime}: \mathbf{B} \rightarrow \mathbf{C}$, if $g h=g^{\prime} h$ then $g=g^{\prime}$. That is, $h$ is right-cancellable in compositions with $\mathcal{K}$-morphisms. Of course every surjective homomorphism is an epimorphism, but the converse is not true. Revisiting the example above, the inclusion of the three-element chain $\mathbf{A}$ into $\mathbf{2} \times \mathbf{2}$ is a $\mathcal{D}_{01}$-epimorphism. The connection between epimorphisms and dominions is that $h: \mathbf{A} \rightarrow \mathbf{B}$ is a $\mathcal{K}$-epimorphism if and only if $\operatorname{dom}_{\mathbf{B}}^{\mathcal{K}} h(\mathbf{A})=\mathbf{B}$.

A class $\mathcal{K}$ is said to have surjective epimorphisms if every $\mathcal{K}$-epimorphism between members of $\mathcal{K}$ is surjective. Although this property is of a categorical nature it has an interesting connection with logic. When $\mathcal{K}$ is the algebraic counterpart of an algebraizable logic $\vdash$ then: $\mathcal{K}$ has surjective epimorphisms if and only if $\vdash$ has the (infinite) Beth property ([2, Thm. 3.17]). For a thorough account on the Beth property in algebraic logic see [2].

Another work we want to mention in regard to the interplay between dominions and the Beth definability property is [4]. In this article the author introduces a family of interpolation properties for a quasivariety $\mathcal{Q}$, in analogy with the Projective Beth Property of propositional logics (see Definition 13 below). The properties defined amount to certain p.p. definable functions being interpolated by terms in $\mathcal{Q}$. Budkin establishes equivalences between these properties and the fact that dominions of certain algebras in $\mathcal{Q}$ are trivial.

The paper is organized as follows. In the next section we establish our notation and present the basic definitions. Section 3 contains our characterization of dominions (Theorem 3), the main result of this article. In Section 4 we show how well-known characterizations of dominions, e.g., Isbell's Zizag Theorem, can be restated in terms of primitive positive definable functions. In Section 5 we introduce Budkin's algebraic Beth Properties, and provide a short proof of the equivalence linking them to dominions. In Section 6 we study the problem of checking a quasivariety for non-surjective epimorphisms. We prove that under certain assumptions it suffices to check in a subclass of the quasivariety. An 
interesting application of these results is that if $\mathcal{F}$ is a finite set of finite algebras with a common near-unanimity term, then it is decidable whether the quasivariety generated by $\mathcal{F}$ has surjective epimorphisms (see Corollary 20).

§2. Preliminaries and Notation. Let $\mathcal{L}$ be a first order language and $\mathcal{K}$ a class of $\mathcal{L}$-structures. We write $\mathbb{I}, \mathbb{S}, \mathbb{H}, \mathbb{P}$ and $\mathbb{P}_{u}$ to denote the class operators for isomorphisms, substructures, homomorphic images, products and ultraproducts, respectively. We write $\mathbb{V}(\mathcal{K})$ for the variety generated by $\mathcal{K}$, that is $\mathbb{H} \mathbb{S P}(\mathcal{K})$; and with $\mathbb{Q}(\mathcal{K})$ we denote the quasivariety generated by $\mathcal{K}$, i.e., $\mathbb{I S P P}_{u}(\mathcal{K})$. If $\mathbf{A}$ is a structure and $S \subseteq A$, let $\langle S\rangle^{\mathbf{A}}$ denote the substructure of $\mathbf{A}$ generated by $S$. We usually abbreviate a sequence $a_{1}, \ldots, a_{n}$ as $\bar{a}$, whenever the length of the sequence is understood or is not relevant. We write $\bar{a} \in A$ to indicate that each member of the sequence is in $A$.

Definition $1\left([13,3]^{1}\right)$. Let $\mathbf{A} \leq \mathbf{B}$ be structures, and $\mathcal{K}$ a class of structures.

- We say that $\mathbf{A}$ dominates an element $b \in B$ relative to $\mathcal{K}$ if for all $\mathbf{C} \in \mathcal{K}$ and all homomorphisms $g, g^{\prime}: \mathbf{B} \rightarrow \mathbf{C}$ such that $\left.g\right|_{A}=\left.g^{\prime}\right|_{A}$, we have $g b=g^{\prime} b$.

- The dominion of $\mathbf{A}$ in $\mathbf{B}$ relative to $\mathcal{K}$ is the set

$$
\operatorname{dom}_{\mathbf{B}}^{\mathcal{K}} \mathbf{A}:=\{b \in B \mid \mathbf{A} \text { dominates } b \text { relative to } \mathcal{K}\} .
$$

Observe that if $\mathbf{A}$ dominates $b$ relative to $\mathcal{K}$, then $\mathbf{A}$ dominates $b$ relative to the classes $\mathbb{I}(\mathcal{K}), \mathbb{S}(\mathcal{K})$ and $\mathbb{P}(\mathcal{K})$. Thus, if $\mathcal{K}$ is closed under ultraproducts and A dominates $b$ relative to $\mathcal{K}$, then $\mathbf{A}$ dominates $b$ relative to $\mathbb{Q}(\mathcal{K})$.

§3. Main Theorem. Recall that a primitive positive (p.p. for brevity) formula is one of the form $\exists \bar{y} \alpha(\bar{x}, \bar{y})$ with $\alpha(\bar{x}, \bar{y})$ a finite conjunction of atomic formulas. We shall need the following fact.

Lemma 2. ([10, Thm. 6.5.7]) Let $\mathbf{A}, \mathbf{B}$ be $\mathcal{L}$-structures. The following are equivalent:

1. Every primitive positive $\mathcal{L}$-sentence that holds in $\mathbf{A}$ holds in $\mathbf{B}$.

2. There is a homomorphism from $\mathbf{A}$ into an ultrapower of $\mathbf{B}$.

Let $\mathcal{K}$ be a class of $\mathcal{L}$-structures. We say that the $\mathcal{L}$-formula $\varphi\left(x_{1}, \ldots x_{n}, y_{1}, \ldots, y_{m}\right)$ defines a function in $\mathcal{K}$ if

$$
\mathcal{K} \vDash \forall \bar{x}, \bar{y}, \bar{z} \varphi(\bar{x}, \bar{y}) \wedge \varphi(\bar{x}, \bar{z}) \rightarrow \bigwedge_{j=1}^{m} y_{j}=z_{j} .
$$

In that case, for each $\mathbf{A} \in \mathcal{K}$ we write $[\varphi]^{\mathbf{A}}$ to denote the $n$-ary partial function defined by $\varphi$ in $\mathbf{A}$. The reader should be aware that throughout this note definable functions are partial functions unless otherwise stated.

If $X$ is a set disjoint with $\mathcal{L}$, we write $\mathcal{L}_{X}$ to denote the language obtained by adding the elements in $X$ as new constant symbols to $\mathcal{L}$. If $\mathbf{B}$ is an $\mathcal{L}$-structure and $A$ is a subset of $B$, let $\mathbf{B}_{A}$ be the expansion of $\mathbf{B}$ to $\mathcal{L}_{A}$ where each new constant names itself. If $\mathcal{L} \subseteq \mathcal{L}^{+}$and $\mathbf{A}$ is an $\mathcal{L}^{+}$-model, let $\left.\mathbf{A}\right|_{\mathcal{L}}$ denote the reduct of $\mathbf{A}$ to $\mathcal{L}$.

Next we present the main result of this article. 
THEOREM 3. Let $\mathcal{L}$ be a first order language and $\mathcal{K}$ a class of $\mathcal{L}$-models closed under ultraproducts. Let $\mathbf{A} \leq \mathbf{B}$ be $\mathcal{L}$-structures and $b \in B$. The following are equivalent:

1. $b \in \operatorname{dom}_{\mathbf{B}}^{\mathcal{K}} \mathbf{A}$.

2. There are a primitive positive formula $\varphi(\bar{x}, y)$ and $\bar{a} \in A$ such that:

(a) $\varphi(\bar{x}, y)$ defines a function in $\mathcal{K}$

(b) $[\varphi]^{\mathbf{B}}(\bar{a})=b$.

Proof. $(1) \Rightarrow(2)$. We can assume that $\mathcal{K}$ is axiomatizable (replacing $\mathcal{K}$ by $\mathbb{I} \mathbb{S}(\mathcal{K})$ if necessary). Suppose $b \in \operatorname{dom}_{\mathbf{B}}^{\mathcal{K}} \mathbf{A}$. Define

$$
\Sigma(x):=\left\{\varphi(x) \mid \varphi(x) \text { is a p.p. formula of } \mathcal{L}_{A} \text { and } \mathbf{B}_{A} \vDash \varphi(b)\right\},
$$

Let $c, d$ be two new constant symbols and take

$$
\mathcal{K}^{*}:=\left\{\mathbf{M} \mid \mathbf{M} \text { is a } \mathcal{L}_{A} \cup\{c, d\} \text {-model and }\left.\mathbf{M}\right|_{\mathcal{L}} \in \mathcal{K}\right\} .
$$

Let $\mathbf{C}$ be a model of $\mathcal{K}^{*}$ such that $\mathbf{C} \vDash \Sigma(c) \cup \Sigma(d)$. By Lemma 2, there are elementary extensions $\mathbf{E}, \mathbf{E}^{\prime}$ of $\mathbf{C}$ and homomorphisms

$$
\begin{aligned}
h: \mathbf{B}_{A} & \left.\rightarrow \mathbf{E}\right|_{\mathcal{L}_{A}} \\
h^{\prime}: \mathbf{B}_{A} & \left.\rightarrow \mathbf{E}^{\prime}\right|_{\mathcal{L}_{A}}
\end{aligned}
$$

such that $h(b)=c^{\mathbf{C}}$ and $h^{\prime}(b)=d^{\mathbf{C}}$. The elementary amalgamation theorem [10, Thm. 6.4.1] provides us with a model $\mathbf{D}$ and elementary embeddings $g$ : $\mathbf{E} \rightarrow \mathbf{D}, g^{\prime}: \mathbf{E}^{\prime} \rightarrow \mathbf{D}$ such that $g$ and $g^{\prime}$ agree on $C$. Next, observe that

$$
\begin{aligned}
g h: \mathbf{B} & \left.\rightarrow \mathbf{D}\right|_{\mathcal{L}} \\
g^{\prime} h^{\prime}: \mathbf{B} & \left.\rightarrow \mathbf{D}\right|_{\mathcal{L}}
\end{aligned}
$$

are homomorphisms that agree on $A$, and since $\left.\mathbf{D}\right|_{\mathcal{L}} \in \mathcal{K}$ we must have

$$
g h(b)=g^{\prime} h^{\prime}(b) .
$$

That is $g\left(c^{\mathbf{C}}\right)=g^{\prime}\left(d^{\mathbf{C}}\right)$. So, as $g$ is $1-1$, and $g$ and $g^{\prime}$ are the same on $C$ we have $c^{\mathbf{C}}=d^{\mathbf{C}}$.

Thus we have shown

$$
\mathcal{K}^{*} \vDash \bigwedge(\Sigma(c) \cup \Sigma(d)) \rightarrow c=d .
$$

By compactness (and using that the conjunction of p.p. formulas is equivalent to a p.p. formula), there is single p.p. $\mathcal{L}$-formula $\varphi(\bar{x}, y)$ such that

$$
\mathcal{K}^{*} \vDash \varphi(\bar{a}, c) \wedge \varphi(\bar{a}, d) \rightarrow c=d,
$$

and hence

$$
\mathcal{K} \vDash \forall \bar{x}, y, z \varphi(\bar{x}, z) \wedge \varphi(\bar{x}, z) \rightarrow y=z .
$$

This completes the proof of $(1) \Rightarrow(2)$.

$(2) \Rightarrow(1)$. Suppose (2) holds. Let $\mathbf{C} \in \mathcal{K}$ and $h, h^{\prime}: \mathbf{B} \rightarrow \mathbf{C}$ homomorphisms agreeing on $A$. Since $\mathbf{B} \vDash \varphi(\bar{a}, b)$, and p.p. formulas are preserved under homomorphisms, we have

$$
\mathbf{C} \vDash \varphi(h \bar{a}, h b) \wedge \varphi\left(h^{\prime} \bar{a}, h^{\prime} b\right) .
$$

Now, $\varphi(\bar{x}, y)$ defines a function in $\mathcal{K}$, so $h \bar{a}=h^{\prime} \bar{a}$ implies $h b=h^{\prime} b$. 
As an immediate application of Theorem 3 we obtain a bound on the size of dominions.

Corollary 4. Let $\mathcal{K}$ be a class of $\mathcal{L}$-structures closed under ultraproducts. For any pair of $\mathcal{L}$-structures $\mathbf{A} \leq \mathbf{B}$ we have

$$
\left|\operatorname{dom}_{\mathbf{B}}^{\mathcal{K}} \mathbf{A}\right| \leq|\mathbf{A}|+|\mathcal{L}|+\aleph_{0}
$$

Assume $\mathcal{K}$ is closed under isomorphisms. A structure $\mathbf{A} \in \mathcal{K}$ is algebraically closed in $\mathcal{K}$ if for every $\mathbf{B} \in \mathcal{K}$ such that $\mathbf{A} \leq \mathbf{B}$, every p.p. formula $\varphi(\bar{x})$ and all $\bar{a} \in A$ we have

$$
\mathbf{B} \vDash \varphi(\bar{a}) \Longrightarrow \mathbf{A} \vDash \varphi(\bar{a}) .
$$

Replacing primitive positive by existential we obtain the definition of existentially closed. Here is another direct consequence of Theorem 3.

Corollary 5. If $\mathbf{A}$ is algebraically closed in $\mathcal{K}$, then $\operatorname{dom}_{\mathbf{B}}^{\mathcal{K}} \mathbf{A}=A$ for every $\mathbf{B} \in \mathcal{K}$ such that $\mathbf{A} \leq \mathbf{B}$.

It is worth noting that $(2) \Rightarrow(1)$ in Theorem 3 always holds, i.e., it does not require $\mathcal{K}$ to be closed under ultraproducts. On the other hand, as the upcoming example shows, the implication $(1) \Rightarrow(2)$ may fail if $\mathcal{K}$ is not closed under ultraproducts.

ExAmple 6 . Let $\mathcal{L}=\{s, 0\}$ where $s$ is a binary function symbol and 0 a constant. Let $\mathbf{B}$ be the $\mathcal{L}$-structure with universe $\omega \cup\{\omega\}$ such that $0^{\mathbf{B}}=0$ and

$$
s^{\mathbf{B}}(a, b)= \begin{cases}0 & \text { if } b=a+1, \\ 1 & \text { otherwise }\end{cases}
$$

and set $\mathcal{K}:=\mathbb{I}(\mathbf{B})$. Take $\mathbf{A}$ as the submodel of $\mathbf{B}$ with universe $\omega$. It is easy to see that the identity is the only endomorphism of $\mathbf{B}$. Thus, in particular, we have that $\operatorname{dom}_{\mathbf{B}}^{\{\mathbf{B}\}} \mathbf{A}=B$. We prove next that there is no p.p. formula with parameters from $A$ defining the element $\omega$ in B. Take $\mathcal{L}^{+}:=\mathcal{L}_{B} \cup\left\{\omega^{\prime}\right\}$, where $\omega^{\prime}$ is a new constant, and let $\Gamma$ be the $\mathcal{L}^{+}$-theory obtained by adding to the elementary diagram of $\mathbf{B}$ the following sentences:

$$
\left\{s\left(n, \omega^{\prime}\right)=1 \mid n \in \omega\right\} \cup\left\{s\left(\omega^{\prime}, n\right)=1 \mid n \in \omega\right\} \cup\left\{\omega \neq \omega^{\prime}\right\} .
$$

It is a routine task to show that $\Gamma$ is consistent. Fix a model $\mathbf{C}$ of $\Gamma$ and define $h, h^{\prime}: \mathbf{B} \rightarrow \mathbf{C}$ by $h(n)=h^{\prime}(n)=n^{\mathbf{C}}$ for all $n \in \omega, h(\omega)=\omega^{\mathbf{C}}$ and $h^{\prime}(\omega)=\omega^{\prime \mathbf{C}}$. Again, it is easy to see that $h$ and $h^{\prime}$ are homomorphisms from $\mathbf{B}$ to $\left.\mathbf{C}\right|_{\mathcal{L}}$. Since they agree on $A$ and $h(\omega) \neq h^{\prime}(\omega)$, we conclude that there is no p.p. formula with parameters from $A$ defining $\omega$ in $\mathbf{B}$.

For some well-known classes $\mathcal{K}$, it turns out that given any pair of structures $\mathbf{A} \leq \mathbf{B}$, the domain of $\mathbf{A}$ in $\mathbf{B}$ relative to $\mathcal{K}$ is generated by functions which are definable by conjunctions of atomic formulas (rather than by p.p. formulas). This is the case, for instance, when $\mathcal{K}$ is the class of distributive lattices (see Section 4.1 below). It is thus natural to ask whether Theorem 3 still holds if the formula in (2) is a conjunction of atomic formulas instead of p.p.. Consider the following condition. 
(3) There is a finite conjunction of atomic formulas $\alpha(\bar{x}, \bar{y})$, and there are elements $a_{1}, \ldots, a_{n} \in A$ and $b_{2}, \ldots, b_{m} \in B$, with $m \geq 1$, such that

(a) $\alpha(\bar{x}, \bar{y})$ defines a function in $\mathcal{K}$

(b) $[\alpha]^{\mathbf{B}}(\bar{a})=\left(b, b_{2}, \ldots, b_{m}\right)$.

It is easily seen that (3) implies (2) of Theorem 3. Our next example shows that (2) does not necessarily imply (3). Hence, we conclude that the statement of Theorem 3 cannot be improved in regard to the kind of formulas involved.

ExAmPle 7. Let $\mathbf{B}$ be the Browerian algebra whose lattice reduct is depicted in Figure 3.1, and let $\mathbf{A}$ be the subalgebra of $\mathbf{B}$ with universe $\left\{a_{0}, a_{1}, \ldots\right\} \cup\{\top\}$. It is proved in [1, Thm. 6.1] that $\operatorname{dom}_{\mathbf{B}}^{\mathbb{V}(\mathbf{B})} \mathbf{A}=B$. Fix $d_{1} \in B \backslash A$, and suppose there are a conjunction of equations $\alpha\left(x_{1}, \ldots, x_{n}, y_{1}, \ldots, y_{m}\right), c_{1}, \ldots, c_{n} \in A$ and $d_{2}, \ldots, d_{m} \in B$ such that

- $\alpha(\bar{x}, \bar{y})$ defines a function in $\mathbb{V}(\mathbf{B})$

- $\mathbf{B} \vDash \alpha(\bar{c}, \bar{d})$.

Let $\mathbf{C}$ and $\mathbf{D}$ be the subalgebras of $\mathbf{B}$ generated by $\bar{c}$ and $\bar{c}, \bar{d}$ respectively. Note that $\mathbf{D}$ is finite and $\mathbf{C}<\mathbf{D}$. Also note that $\alpha(\bar{x}, \bar{y})$ defines a function in $\mathbb{V}(\mathbf{D})$, and $\mathbf{D} \vDash \alpha(\bar{c}, \bar{d})$, because $\alpha$ is quantifier-free. So we have $\operatorname{dom}_{\mathbf{D}}^{\mathbb{V}(\mathbf{D})} \mathbf{C}=D$; but this is not possible, as Corollary 5.5 in [1] says that epimorphisms are surjective in finitely generated varieties of Browerian algebras.

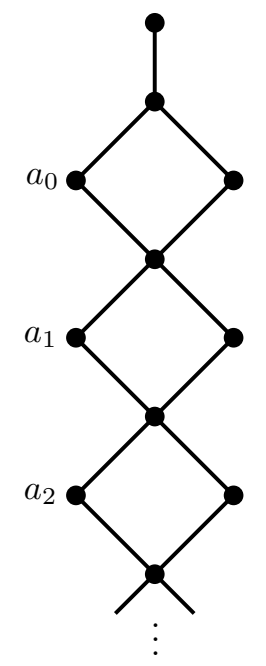

Figure 3.1

§4. Generating Sets. Theorem 3 suggests that a way to characterize dominions in a given class $\mathcal{K}$ is to find a concise set of p.p. formulas that suffices to generate dominions in $\mathcal{K}$. This motivates the following definition. We say that a set $\Gamma$ of formulas generates dominions in $\mathcal{K}$ provided that each formula in $\Gamma$ defines a function in $\mathcal{K}$, and for each $\mathbf{A} \leq \mathbf{B}$ from $\mathcal{K}$ we have

$$
\operatorname{dom}_{\mathbf{B}}^{\mathcal{K}} \mathbf{A}=\langle A\rangle^{\left\langle\mathbf{B},[\varphi]^{\mathbf{B}}\right\rangle_{\varphi \in \Gamma} .}
$$


That is, $\operatorname{dom}_{\mathbf{B}}^{\mathcal{K}} \mathbf{A}$ is the smallest subset of $B$ including $A$, closed under the fundamental operations of $\mathbf{B}$ and closed under the functions defined in $\mathbf{B}$ by formulas from $\Gamma$.

Below we take a look at two classes whose dominions are well understood, and recast their characterization results in terms of generating sets.

4.1. Distributive lattices. Let $\mathcal{D}$ be the variety of distributive lattices, and let $\mathbf{B}$ in $\mathcal{D}$. Given elements $a \leq b \leq c$ in $B$, recall that a relative complement of $b$ in the interval $[a, c]$ is an element $d \in B$ such that $b \wedge d=a$ and $b \vee d=c$. It is well known that in a distributive lattice every element has at most one complement in each interval it belongs to. A subset $A$ of $B$ is said to be closed under relative complementation provided that for any $a \leq b \leq c$ in $A$, if $d \in B$ is the relative complement of $b$ in the interval $[a, c]$, then $d \in A$. Dominions in $\mathcal{D}$ are characterized by the following.

Theorem 8 ([19, Thm 2.4]). Let $\mathbf{A} \leq \mathbf{B}$ be distributive lattices. Then $\operatorname{dom}_{\mathbf{B}}^{\mathcal{D}} \mathbf{A}$ is the smallest sublattice of $\mathbf{B}$ that contains $A$ and is closed under relative complementation.

Define

$$
\varphi_{\mathrm{rc}}\left(x_{1}, x_{2}, x_{3}, y\right):=\left(x_{2} \wedge y=x_{1}\right) \&\left(x_{2} \vee y=x_{3}\right) .
$$

Note that for $\mathbf{B}$ in $\mathcal{D}$ and $a, b, c, d \in B$ we have $\mathbf{B} \vDash \varphi_{\mathrm{rc}}(a, b, c, d)$ iff $a \leq b \leq c$ and $d$ is the relative complement of $b$ in the interval $[a, c]$. The fact that relative complements are unique in distributive lattices ensures that $\varphi_{\mathrm{rc}}$ defines a function in $\mathcal{D}$. Thus we have:

Corollary 9. The set $\left\{\varphi_{\mathrm{rc}}\right\}$ generates dominions in $\mathcal{D}$.

4.2. Semigroups. Let $\mathcal{S}$ denote the variety of semigroups. One of the most influential results on dominions is the following.

Theorem 10 (Isbell's Zigzag Theorem [13, Thm 2.3]). Let $\mathbf{A} \leq \mathbf{B}$ be semigroups, and let $b \in B$. Then, $b \in \operatorname{dom}_{\mathbf{B}}^{\mathcal{S}} \mathbf{A}$ iff there are an integer $m \geq 1$, $a_{0}, \ldots, a_{2 m} \in A$ and $b_{1}, \ldots, b_{m}, c_{1}, \ldots, c_{m} \in B$ such that the following equalities hold:

$$
\begin{aligned}
b & =a_{0} c_{1} \\
b & =b_{m} a_{2 m} \\
a_{0} & =b_{1} a_{1} \\
a_{2 i-1} c_{i} & =a_{2 i} c_{i+1} \quad \text { for } 1 \leq i \leq m-1 \\
b_{i} a_{2 i} & =b_{i+1} a_{2 i+1} \quad \text { for } 1 \leq i \leq m-1 \\
a_{2 m-1} c_{m} & =a_{2 m} .
\end{aligned}
$$

This theorem has received quite some attention in the literature (see e.g. [9, $11,12,15,16,17])$. We shall restate it as result on generating sets. For each integer $m \geq 1$ let $\alpha_{m}\left(x_{0}, \ldots, x_{2 m}, y, z_{1}, \ldots, z_{m}, w_{1}, \ldots, w_{m}\right)$ be the conjunction 
of the following atomic formulas

$$
\begin{aligned}
y & =x_{0} w_{1} \\
y & =z_{m} x_{2 m} \\
x_{0} & =z_{1} x_{1} \\
x_{2 i-1} w_{i} & =x_{2 i} w_{i+1} \quad \text { for } 1 \leq i \leq m-1 \\
z_{i} x_{2 i} & =z_{i+1} x_{2 i+1} \quad \text { for } 1 \leq i \leq m-1 \\
x_{2 m-1} w_{m} & =x_{2 m} .
\end{aligned}
$$

Now define

$$
\zeta_{m}\left(x_{0}, \ldots, x_{2 m}, y\right):=\exists \bar{z} \bar{w} \alpha_{m}(\bar{x}, y, \bar{z}, \bar{w}) .
$$

Lemma 11. For each integer $m \geq 1$ the formula $\zeta_{m}\left(x_{0}, \ldots, x_{2 m}, y\right)$ defines a function in $\mathcal{S}$.

Proof. Let $\mathbf{A} \in \mathcal{S}$ and suppose there are $\bar{a}, b, b^{\prime} \in A$ such that $\mathbf{A} \vDash \zeta_{m}(\bar{a}, b) \wedge$ $\zeta_{m}\left(\bar{a}, b^{\prime}\right)$; we prove that $b=b^{\prime}$. There are $\bar{b}, \bar{c}, \bar{b}^{\prime}, \bar{c}^{\prime} \in A$ such that $\mathbf{A} \vDash$ $\alpha_{m}(\bar{a}, b, \bar{b}, \bar{c}) \wedge \alpha_{m}\left(\bar{a}, b^{\prime}, \overline{b^{\prime}}, \bar{c}^{\prime}\right)$. So we have

$$
\begin{aligned}
b & =a_{0} c_{1} \\
& =b_{1}^{\prime} a_{1} c_{1} \\
& =b_{1}^{\prime} a_{2} c_{2} \\
& =b_{2}^{\prime} a_{3} c_{2} \\
& \vdots \\
& =b_{m}^{\prime} a_{2 m-1} c_{m} \\
& =b_{m}^{\prime} a_{2 m} \\
& =b^{\prime} .
\end{aligned}
$$

Corollary 12. The set $\left\{\zeta_{m} \mid m \geq 1\right\}$ generates dominions in $\mathcal{S}$.

§5. Dominions and Projective Beth Properties. In the article [4] A. Budkin defines a family of interpolation properties for a quasivariety $\mathcal{Q}$, in analogy with the Projective Beth Property of propositional logics. The properties introduced amount to certain p.p. definable functions being interpolated by terms in $\mathcal{Q}$. The main point of [4] is to establish equivalences between the interpolation properties and the fact that dominions of certain algebras in $\mathcal{Q}$ are trivial. In this section we show how these equivalences are obtained as direct applications of Theorem 3. First we need some definitions.

Definition 13 ([4]). Let $\mathcal{L}$ be an algebraic language, and let $\mathcal{Q}$ be a quasivariety of $\mathcal{L}$-algebras. Let $\bar{x}$ be a possibly infinite sequence of pairwise distinct variables, let $\Delta(\bar{x})$ be a set of term-equalities in $\bar{x}$, and let $n \geq 1$ be an integer.

- $\mathcal{Q}$ has the projective property $\operatorname{PBP}_{n}(\Delta)$ if for every set of atomic formulas $\Gamma\left(\bar{x}, q_{1}, \ldots, q_{n}, y\right)$ and every term $f(\bar{x}, \bar{q})$ such that

(i) $\mathcal{Q} \vDash \Delta(\bar{x}) \cup \Gamma\left(\bar{x}, q_{1}, \ldots, q_{n}, y\right) \cup \Gamma\left(\bar{x}, q_{1}^{\prime}, \ldots, q_{n}^{\prime}, z\right) \rightarrow y=z$ 
(ii) $\mathcal{Q} \vDash \Delta(\bar{x}) \cup \Gamma(\bar{x}, \bar{q}, y) \rightarrow y=f(\bar{x}, \bar{q})$,

there is an $\mathcal{L}$-term $t(\bar{x})$ satisfying

$$
\mathcal{Q} \vDash \Delta(\bar{x}) \cup \Gamma(\bar{x}, \bar{q}, y) \rightarrow y=t(\bar{x}) .
$$

- $\mathbf{A} \in \mathcal{Q}$ is $n$-closed in $\mathcal{Q}$ provided that for all $\mathbf{B} \in \mathcal{Q}$ such that $\mathbf{A} \leq \mathbf{B}$ and $\mathbf{B}=\left\langle A \cup\left\{b_{1}, \ldots, b_{n}\right\}\right\rangle$ for some $b_{1}, \ldots, b_{n} \in B$, we have $\operatorname{dom}_{\mathbf{B}}^{\mathcal{Q}} \mathbf{A}=A$.

- $\mathbf{A} \in \mathcal{Q}$ is $\mathbf{H}_{n}$-closed in $\mathcal{Q}$ if every homomorphic image of $\mathbf{A}$ in $\mathcal{Q}$ is $n$-closed in $\mathcal{Q}$.

Let $\mathbf{F}_{\mathcal{Q}}(\bar{x})$ be the $\mathcal{Q}$-free algebra, freely generated by $\bar{x}$. We write $\mathbf{F}_{\mathcal{Q}}(\bar{x}) / \Delta(\bar{x})$ to denote the quotient of $\mathbf{F}_{\mathcal{Q}}(\bar{x})$ by the $\mathcal{Q}$-congruence generated by $\{(t, s) \mid t=$ $s \in \Delta(\bar{x})\}$.

TheOrem $14\left(\left[4\right.\right.$, Thm. 3]). A quasivariety $\mathcal{Q}$ has the projective property $P B P_{n}(\Delta)$ if and only if $\mathbf{F}_{\mathcal{Q}}(\bar{x}) / \Delta(\bar{x})$ is $\mathbf{H}_{n}$-closed in $\mathcal{Q}$.

Proof. For the right-to-left direction see [4, Thm. 3]. Suppose $\mathcal{Q}$ has the $P B P_{n}(\Delta)$. Let $\mathbf{A} \leq \mathbf{B}=\left\langle A \cup\left\{b_{1}, \ldots, b_{n}\right\}\right\rangle \in \mathcal{Q}$ with $\mathbf{A}$ a homomorphic image of $\mathbf{F}_{\mathcal{Q}}(\bar{x}) / \Delta(\bar{x})$, and take $b \in \operatorname{dom}_{\mathbf{B}}^{\mathcal{Q}} \mathbf{A}$. We prove that $b \in A$. Let $\bar{a} \in$ $A$ such that $\bar{a}$ generates $\mathbf{A}$ and $\mathbf{A} \vDash \Delta(\bar{a})$. By Theorem 3 there are a p.p. formula $\varphi(\bar{v}, y)=\exists w_{1} \ldots w_{m} \bigwedge_{i=1}^{l} \gamma_{i}\left(v_{1}, \ldots, v_{k}, \bar{w}, y\right)$ defining a function in $\mathcal{Q}$, and terms $t_{1}(\bar{x}), \ldots, t_{k}(\bar{x})$ such that $[\varphi]^{\mathbf{B}}\left(t_{1}^{\mathbf{A}}(\bar{a}), \ldots, t_{k}^{\mathbf{A}}(\bar{a})\right)=b$. Take terms $s_{1}(\bar{x}, \bar{q}), \ldots, s_{m}(\bar{x}, \bar{q}), f(\bar{x}, \bar{q})$ such that

$$
\mathbf{B} \vDash \bigwedge_{i=1}^{l} \gamma_{i}\left(t_{1}(\bar{a}), \ldots, t_{k}(\bar{a}), s_{1}(\bar{a}, \bar{b}), \ldots, s_{m}(\bar{a}, \bar{b}), f(\bar{a}, \bar{b})\right),
$$

where $\bar{b}$ is $b_{1}, \ldots, b_{n}$. Now define $\Gamma\left(\bar{x}, q_{1}, \ldots, q_{n}, y\right)$ as the set

$$
\left\{\gamma_{i}\left(t_{1}(\bar{x}), \ldots, t_{k}(\bar{x}), s_{1}(\bar{x}, \bar{q}), \ldots, s_{m}(\bar{x}, \bar{q}), y\right) \mid i \in\{1, \ldots, l\}\right\} \cup\{y=f(\bar{x}, \bar{q})\},
$$

and note that (i) and (ii) of Definition 13 hold for $\Gamma\left(\bar{x}, q_{1}, \ldots, q_{n}, y\right)$ and $f(\bar{x}, \bar{q})$. So, there is a term $t(\bar{x})$ such that $\mathcal{Q} \vDash \Delta(\bar{x}) \cup \Gamma(\bar{x}, \bar{q}, y) \rightarrow y=t(\bar{x})$, and hence $b=t^{\mathbf{B}}(\bar{a}) \in A$.

Budkin also defines the projective property $P B P(\Delta)$ where no restriction is posed on the length of $\bar{q}$ and the requirement (ii) is dropped. The characterization of the $\operatorname{PBP}(\Delta)$ in terms of dominions [4, Thm. 2] can be obtained from Theorem 3 with a proof similar to the one above.

§6. Epimorphisms and epic substructures. Let A, B be structures, and $\mathcal{K}$ a class of structures.

- A homomorphism $h: \mathbf{A} \rightarrow \mathbf{B}$ is a $\mathcal{K}$-epimorphism if for every $\mathbf{C} \in \mathcal{K}$ and homomorphisms $g, g^{\prime}: \mathbf{B} \rightarrow \mathbf{C}$, if $g h=g^{\prime} h$ then $g=g^{\prime}$.

It follows at once from the definitions that $h: \mathbf{A} \rightarrow \mathbf{B}$ is a $\mathcal{K}$-epimorphism iff $\operatorname{dom}_{\mathbf{B}}^{\mathcal{K}} h(\mathbf{A})=B$.

- A class $\mathcal{K}$ has surjective epimorphisms if for all $\mathbf{A}, \mathbf{B} \in \mathcal{K}$ every $\mathcal{K}$-epimorphism from $\mathbf{A}$ to $\mathbf{B}$ is surjective. 
As mentioned in Section 1, this property is of special interest to algebraic logic, since whenever $\mathcal{K}$ is the algebraic counterpart of a logic $\vdash$, then $\mathcal{K}$ has surjective epimorphisms if and only if $\vdash$ has the the (infinite) Beth property [2, Thm. 3.17].

When considering whether epimorphisms are surjective in a class $\mathcal{K}$ the notion of dominion proves very helpful. This is because it translates a categorical problem into an algebraic (or model theoretic) problem, and even more so in the light of Theorem 3. The following definition isolates the phenomenon we want to investigate.

- $\mathbf{A}$ is an epic substructure of $\mathbf{B}$ with respect to $\mathcal{K}$ if $\mathbf{A} \leq \mathbf{B}$ and $\operatorname{dom}_{\mathbf{B}}^{\mathcal{K}} \mathbf{A}=B$. Notation: $\mathbf{A} \leq_{e}^{\mathcal{K}} \mathbf{B}$.

We say that $\mathbf{A}$ is a proper epic substructure of $\mathbf{B}$ with respect to $\mathcal{K}$, and write $\mathbf{A}<_{e}^{\mathcal{K}} \mathbf{B}$, if $\mathbf{A} \leq_{e}^{\mathcal{K}} \mathbf{B}$ and $\mathbf{A} \neq \mathbf{B}$.

The next lemma states the straightforward connection between epic substructures and epimorphisms.

Lemma 15. Suppose $h: \mathbf{A} \rightarrow \mathbf{B}$. The following are equivalent:

1. $h$ is a $\mathcal{K}$-epimorphism.

2. The inclusion map $\iota: h(\mathbf{A}) \rightarrow \mathbf{B}$ is a $\mathcal{K}$-epimorphism.

3. $h(\mathbf{A}) \leq_{e}^{\mathcal{K}} \mathbf{B}$.

Here are some easy facts used in the sequel.

Lemma 16. Let $\mathbf{A}, \mathbf{B}, \mathbf{C} \in \mathcal{K}$.

1. $\mathbf{A} \leq_{e}^{\mathcal{K}} \mathbf{B}$ if and only if $\mathbf{A} \leq_{e}^{\mathbb{I S P}(\mathcal{K})} \mathbf{B}$.

2. If $\mathbf{A} \leq_{e}^{\mathcal{K}} \mathbf{B}$ and $h: \mathbf{B} \rightarrow \mathbf{C}$, then $h(\mathbf{A}) \leq_{e}^{\mathcal{K}} h(\mathbf{B})$.

3. Let $\mathcal{Q}$ be a quasivariety. The following are equivalent:

(a) $\mathcal{Q}$ has surjective epimorphisms.

(b) For all $\mathbf{A}, \mathbf{B} \in \mathcal{Q}$ we have that $\mathbf{A} \leq_{e}^{\mathcal{Q}} \mathbf{B}$ implies $\mathbf{A}=\mathbf{B}$.

Given a quasivariety $\mathcal{Q}$ it is in a general a difficult problem to determine whether $\mathcal{Q}$ has surjective epimorphisms, or equivalently, no proper epic substructures. Below we prove two results that, under certain assumptions on $\mathcal{Q}$, provide a (hopefully) more manageable class $\mathcal{C} \subseteq \mathcal{Q}$ such that $\mathcal{Q}$ has surjective epimorphisms iff $\mathcal{C}$ has surjective epimorphisms.

Our first result provides such a class $\mathcal{C}$ for quasivarieties with a near-unanimity term. The second one for arithmetical varieties whose class of finitely subdirectly irreducible members is universal.

For the remainder of this section all languages considered are algebraic, i.e., without relation symbols. We frequently use the name algebra for a structure of an algebraic language.

6.1. Quasivarieties with a near-unanimity term. A $k$-ary term $t\left(x_{1}, \ldots, x_{k}\right)$ is a near-unanimity term for the class $\mathcal{K}$ if $k \geq 3$ and $\mathcal{K}$ satisfies the identities

$$
t(x, \ldots, x, y)=t(x, \ldots, x, y, x)=\cdots=t(y, x, \ldots, x)=x .
$$

When $k=3$ the term $t$ is called a majority term for $\mathcal{K}$. In every structure with a lattice reduct the term $(x \vee y) \wedge(x \vee z) \wedge(y \vee z)$ is a majority term. This example is specially relevant since many classes of structures arising from logic have lattice reducts. 
For functions $f_{i}: X_{i} \rightarrow Y_{i}$ with $i \in\{1, \ldots, k\}$ let $\left(f_{1}, \ldots, f_{k}\right): X_{1} \times \cdots \times X_{k} \rightarrow$ $Y_{1} \times \cdots \times Y_{k}$ be defined by $\left(f_{1}, \ldots, f_{k}\right)\left(a_{1}, \ldots, a_{k}\right):=\left(f_{1}\left(a_{1}\right), \ldots, f_{k}\left(a_{k}\right)\right)$.

THEOREM $17([18])$. Let $\mathcal{K}$ be a class of structures with an $k$-ary near-unanimity term and suppose the first-order formula $\varphi(\bar{x}, y)$ defines a function in $\mathcal{K}$. The following are equivalent:

1. There is a term $t(\bar{x})$ such that $\mathcal{K} \vDash \forall \bar{x}, y \varphi(\bar{x}, y) \rightarrow y=t(\bar{x})$.

2. For all $\mathbf{A}_{1}, \ldots, \mathbf{A}_{k} \in \mathbb{P}_{u}(\mathcal{K})$, all $\mathbf{S} \leq \mathbf{A}_{1} \times \cdots \times \mathbf{A}_{k}$ and all $s_{1}, \ldots, s_{n} \in S$ such that $\left([\varphi]^{\mathbf{A}_{1}}, \ldots,[\varphi]^{\mathbf{A}_{k}}\right)(\bar{s})$ is defined, we have that $\left([\varphi]^{\mathbf{A}_{1}}, \ldots,[\varphi]^{\mathbf{A}_{k}}\right)(\bar{s})$ is in $S$.

An algebra $\mathbf{A}$ in the quasivariety $\mathcal{Q}$ is relatively subdirectly irreducible provided its diagonal congruence is completely meet irreducible in the lattice of $\mathcal{Q}$-congruences of $\mathbf{A}$. We write $\mathcal{Q}_{\mathrm{RSI}}$ to denote the class of relatively subdirectly irreducible members of $\mathcal{Q}$. For a class $\mathcal{K}$ and a positive integer $k$ let

$$
\mathbb{P}_{k}(\mathcal{K}):=\left\{\mathbf{A}_{1} \times \cdots \times \mathbf{A}_{k} \mid \mathbf{A}_{1}, \ldots, \mathbf{A}_{k} \in \mathcal{K}\right\} .
$$

THEOREM 18. Let $\mathcal{Q}$ be a quasivariety with a k-ary near-unanimity term and let $\mathcal{M}=\mathbb{P}_{u}\left(\mathcal{Q}_{\mathrm{RSI}}\right)$. The following are equivalent:

1. $\mathcal{Q}$ has surjective epimorphisms.

2. For all $\mathbf{A}, \mathbf{B} \in \mathcal{Q}$ we have that $\mathbf{A} \leq_{e}^{\mathcal{Q}} \mathbf{B}$ implies $\mathbf{A}=\mathbf{B}$.

3. For all $\mathbf{A}, \mathbf{B} \in \mathbb{S P}_{k}(\mathcal{M})$ we have that $\mathbf{A} \leq_{e}^{\mathbb{P}_{k}(\mathcal{M})} \mathbf{B}$ implies $\mathbf{A}=\mathbf{B}$.

4. $\mathbb{S P}_{k}(\mathcal{M})$ has surjective epimorphisms.

Proof. The equivalences $(1) \Leftrightarrow(2)$ and $(3) \Leftrightarrow(4)$ are immediate, and (2) clearly implies (3). We prove (3) $\Rightarrow(2)$. Suppose $\mathbf{A} \leq_{e}^{\mathcal{Q}} \mathbf{B}$ and let $b \in B$. We shall see that $b \in A$. By Theorem 3 there is a p.p. $\mathcal{L}$-formula $\varphi(\bar{x}, y)$ defining a function in $\mathcal{Q}$, and such that $[\varphi]^{\mathrm{B}}(\bar{a})=b$ for some $\bar{a} \in A^{n}$. Let

$$
\Sigma:=\left\{\varepsilon \mid \varepsilon \text { is a p.p. formula of } \mathcal{L}_{A} \text { and } \mathbf{B}_{A} \vDash \varepsilon\right\},
$$

and define

$$
\mathcal{K}:=\left\{\mathbf{C} \in \operatorname{Mod}(\Sigma)|\mathbf{C}|_{\mathcal{L}} \in \mathcal{M}\right\} .
$$

Let $\psi(y):=\varphi(\bar{a}, y)$, and note that $\psi(y)$ defines a nullary function in $\mathcal{K}$. Note as well that $\exists y \psi(y) \in \Sigma$, and hence $[\psi]^{\mathbf{C}}$ is defined for every $\mathbf{C} \in \mathcal{K}$. We aim to apply Theorem 17 to $\mathcal{K}$ and $\psi(y)$. To this end fix $\mathbf{C}_{1}, \ldots, \mathbf{C}_{k} \in \mathbb{P}_{u}(\mathcal{K})=\mathcal{K}$ and let $\mathbf{S} \leq \mathbf{C}_{1} \times \cdots \times \mathbf{C}_{k}$. Note that as $\Sigma$ is a set of p.p. formulas we have $\mathbf{C}_{1} \times \cdots \times \mathbf{C}_{k} \vDash \Sigma$, and thus by Lemma 2 there is an ultrapower $\mathbf{E}$ of $\mathbf{C}_{1} \times \cdots \times \mathbf{C}_{k}$ and a homomorphism $h: \mathbf{B}_{A} \rightarrow \mathbf{E}$. We have that $\mathbf{E} \in \mathbb{P}_{u} \mathbb{P}_{k}(\mathcal{K}) \subseteq \mathbb{P}_{k} \mathbb{P}_{u}(\mathcal{K})=$ $\mathbb{P}_{k}(\mathcal{K})$, and so

$$
\left.\mathbf{E}\right|_{\mathcal{L}} \in \mathbb{P}_{k}\left(\left.\mathcal{K}\right|_{\mathcal{L}}\right) \subseteq \mathbb{P}_{k}(\mathcal{M})
$$

Next observe that since $h(\mathbf{A}) \leq_{e}^{\mathcal{Q}} h(\mathbf{B})$, and $h(\mathbf{A}), h(\mathbf{B}) \leq\left.\mathbf{E}\right|_{\mathcal{L}}$, by $(3)$ it follows that $h(A)=h(B)$. Also, as $\mathbf{S}$ is an $\mathcal{L}_{A}$-subalgebra of $\mathbf{E}$, we have that

$$
h\left(\mathbf{B}_{A}\right)=h\left(\mathbf{A}_{A}\right) \leq \mathbf{S} .
$$

The fact that $\mathbf{B} \vDash \psi(b)$, implies $\mathbf{E} \vDash \psi(h b)$, and so $[\psi]^{\mathbf{E}}=h b \in S$. We know that $\left\{\mathbf{C}_{1}, \ldots, \mathbf{C}_{k}, \mathbf{C}_{1} \times \cdots \times \mathbf{C}_{k}\right\} \vDash \exists y \psi(y)$; furthermore, since $\psi$ is p.p., we have 


$$
\begin{aligned}
& \left([\psi]^{\mathbf{C}_{1}}, \ldots,[\psi]^{\mathbf{C}_{k}}\right)=[\psi]^{\mathbf{C}_{1} \times \cdots \times \mathbf{C}_{k}} \text {. Putting all this together } \\
& \left([\psi]^{\mathbf{C}_{1}}, \ldots,[\psi]^{\mathbf{C}_{k}}\right)=[\psi]^{\mathbf{C}_{1} \times \cdots \times \mathbf{C}_{k}}=[\psi]^{\mathbf{E}} \in S .
\end{aligned}
$$

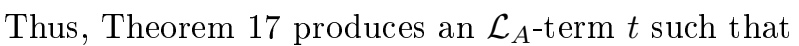

$$
\mathcal{K} \vDash \forall y \psi(y) \rightarrow y=t .
$$

In particular, for all $\mathbf{C} \in \mathcal{Q}_{\mathrm{RSI}}$ and all $c_{1}, \ldots, c_{n} \in \mathbf{C}$ such that $[\varphi]^{\mathbf{C}}(\bar{c})$ is defined, we have

$$
[\varphi]^{\mathbf{C}}(\bar{c})=t^{\mathbf{C}}(\bar{c}) .
$$

Next let $\left\{\mathbf{B}_{i} \mid i \in I\right\} \subseteq \mathcal{Q}_{\mathrm{RSI}}$ such that $\mathbf{B} \leq \prod_{I} \mathbf{B}_{i}$ is a subdirect product. For every $i \in I$ let $\mathbf{B}_{i}^{A}$ be the expansion of $\mathbf{B}_{i}$ to $\mathcal{L}_{A}$ given by $a^{\mathbf{B}_{i}^{A}}=\pi_{i}(a)$, where $\pi_{i}: \mathbf{B} \rightarrow \mathbf{B}_{i}$ is the projection map. It is clear that

$$
\mathbf{B}_{A} \leq \prod_{I} \mathbf{B}_{i}^{A} .
$$

Now, each $\mathbf{B}_{i}^{A}$ is a homomorphic image of $\mathbf{B}_{A}$, so $\mathbf{B}_{i}^{A} \vDash \Sigma$ and thus $\mathbf{B}_{i}^{A} \in \mathcal{K}$ for all $i \in I$. Since $\forall y \psi(y) \rightarrow y=t$ is (equivalent to) a quasi-identity, from (6.1) and (6.2) we have

$$
\mathbf{B}_{A} \vDash \forall y \psi(y) \rightarrow y=t .
$$

Hence $b=t^{\mathbf{B}_{A}} \in A$, and the proof is finished.

Observe that Theorem 18 holds for any $\mathcal{M} \subseteq \mathcal{Q}$ closed under ultraproducts and containing $\mathcal{Q}_{\mathrm{RSI}}$.

COROLlaRY 19. Let $\mathcal{Q}$ be a finitely generated quasivariety with $k$-ary nearunanimity term. The following are equivalent:

1. $\mathcal{Q}$ has surjective epimorphisms.

2. $\operatorname{SP}_{k}\left(\mathcal{Q}_{\mathrm{RSI}}\right)$ has surjective epimorphisms.

Proof. For any class $\mathcal{K}$ we have $\mathbb{Q}(\mathcal{K})_{\text {RSI }} \subseteq \mathbb{I S P}_{u}(\mathcal{K})$. Thus if $\mathcal{Q}$ is finitely generated, then $\mathcal{Q}_{\mathrm{RSI}}$ is (up to isomorphic copies) a finite set of finite algebras, and the corollary follows at once from Theorem 18.

Recall that an algebra $\mathbf{A}$ is finitely subdirectly irreducible if its diagonal congruence is meet irreducible in the congruence lattice of $\mathbf{A}$. It is subdirectly irreducible if the diagonal is completely meet irreducible. For a variety $\mathcal{V}$ we write $\left(\mathcal{V}_{\mathrm{FSI}}\right)$ $\mathcal{V}_{\mathrm{SI}}$ to denote its class of (finitely) subdirectly irreducible members.

The following is an interesting consequence of Corollary 19.

COROLlary 20. Suppose $\mathcal{L}$ is a finite algebraic language. Let $\mathcal{F}$ be a finite set of finite $\mathcal{L}$-algebras with a common $k$-ary near-unanimity term. It is decidable whether the (quasi)variety generated by $\mathcal{F}$ has surjective epimorphisms.

Proof. Let $\mathcal{V}$ be the variety generated by $\mathcal{F}$. By Jónsson's lemma [14] $\mathcal{V}_{\mathrm{SI}} \subseteq$ $\mathbb{H S P P}_{u}(\mathcal{F})=\mathbb{H} \mathbb{S}(\mathcal{F})$ is a finite set of finite structures, and by Corollary 19 it suffices to decide whether $\mathbb{S P}_{k}\left(\mathcal{V}_{\mathrm{SI}}\right)$ has surjective epimorphisms, and this is clearly a decidable problem. If $\mathcal{Q}$ is the quasivariety generated by $\mathcal{F}$, then $\mathcal{Q}_{\mathrm{RSI}} \subseteq \mathbb{I S P}_{u}(\mathcal{F})=\mathbb{I S}(\mathcal{F})$, and the same reasoning applies. 
6.2. Arithmetical varieties whose FSI members form a universal class. A variety $\mathcal{V}$ is arithmetical if for every $\mathbf{A} \in \mathcal{V}$ the congruence lattice of $\mathbf{A}$ is distributive and the join of any two congruences is their composition. For example, the variety of boolean algebras is arithmetical.

LEMMA 21. Let $\mathcal{V}$ be an arithmetical variety such that $\mathcal{V}_{\mathrm{FSI}}$ is a universal class, and let $\varphi(\bar{x}, y)$ be a p.p. formula defining a function in $\mathcal{V}$. Suppose that for all $\mathbf{A} \in \mathcal{V}_{\mathrm{FSI}}$, all $\mathbf{S} \leq \mathbf{A}$ and all $s_{1}, \ldots, s_{n} \in S$ such that $\mathbf{A} \vDash \exists y \varphi(\bar{s}, y)$, we have $\mathbf{S} \vDash \exists y \varphi(\bar{s}, y)$. Then there is a term $t(\bar{x})$ such that $\mathcal{V} \vDash \forall \bar{x}, y \varphi(\bar{x}, y) \rightarrow y=t(\bar{x})$.

Proof. Add new constants $c_{1}, \ldots, c_{n}$ to the language of $\mathcal{V}$ and let $\mathcal{K}:=$ $\left\{(\mathbf{A}, \bar{a}) \mid \mathbf{A} \vDash \exists y \varphi(\bar{c}, y)\right.$ and $\left.\mathbf{A} \in \mathcal{V}_{\mathrm{FSI}}\right\}$. Note that $\psi(y):=\varphi(\bar{c}, y)$ defines a nullary function in $\mathcal{K}$, and this function is defined for every member of $\mathcal{K}$. Also note that by our assumptions $\mathcal{K}$ is a universal class. Using Jónsson's lemma [14] it is not hard to show that $\mathbb{V}(\mathcal{K})_{\mathrm{FSI}}=\mathcal{K}$. Since $\left.\mathcal{K}\right|_{\mathcal{L}}$ is contained in an arithmetical variety it has a Pixley Term [5, Thm. 12.5], which also serves as a Pixley Term for $\mathcal{K}$, and thus $\mathbb{V}(\mathcal{K})$ is arithmetical. Next we show that $\psi(y)$ is equivalent to a positive open formula in $\mathcal{K}$. By [6, Thm. 3.1] it suffices to show that

- For all $\mathbf{A}, \mathbf{B} \in \mathcal{K}$, all $\mathbf{S} \leq \mathbf{A}$, all $h: \mathbf{S} \rightarrow \mathbf{B}$ and every $a \in A$ we have that $\mathbf{A} \vDash \psi(a)$ implies $\mathbf{B} \vDash \psi(h a)$.

So suppose $\mathbf{A} \vDash \psi(a)$. From our hypothesis and the fact that $\psi(y)$ defines a function we have $\mathbf{S} \vDash \psi(a)$, and as $\psi(y)$ is p.p. we obtain $\mathbf{B} \vDash \psi(h a)$. Hence there is a positive open formula $\beta(y)$ equivalent to $\psi(y)$ in $\mathcal{K}$. Now, [7, Thm. $2.3]$ implies that there is a conjunction of equations $\alpha(y)$ equivalent to $\beta(y)$ (and thus to $\psi(y)$ ) in $\mathcal{K}$. We have $\mathcal{K} \vDash \exists$ ! $y \alpha(y)$, and by [6, Lemma 7.8] there is an $\mathcal{L} \cup\left\{c_{1}, \ldots, c_{n}\right\}$-term $t^{\prime}$ such that $\mathbb{V}(\mathcal{K}) \vDash \alpha\left(t^{\prime}\right)$. Let $t\left(x_{1}, \ldots, x_{n}\right)$ be an $\mathcal{L}$-term such that $t^{\prime}=t(\bar{c})$. So, if $\Gamma$ is a set of axioms for $\mathcal{V}_{\mathrm{FSI}}$, we have

$$
\Gamma \cup\{\exists y \varphi(\bar{c}, y)\} \vDash \varphi(\bar{c}, t(\bar{c})),
$$

and this implies

$$
\Gamma \vDash \exists y \varphi(\bar{c}, y) \rightarrow \varphi(\bar{c}, t(\bar{c})),
$$

or equivalently

$$
\mathcal{V}_{\mathrm{FSI}} \vDash \forall y(\varphi(\bar{c}, y) \rightarrow \varphi(\bar{c}, t(\bar{c}))) .
$$

This and the fact that $\varphi(\bar{x}, y)$ defines a function in $\mathcal{V}$ yields

$$
\mathcal{V}_{\mathrm{FSI}} \vDash \forall \bar{x}, y \varphi(\bar{x}, y) \rightarrow y=t(\bar{x}) .
$$

To conclude, note that $\forall \bar{x}, y \varphi(\bar{x}, y) \rightarrow y=t(\bar{x})$ is logically equivalent to a quasi-identity, and since it holds in $\mathcal{V}_{\text {FSI }}$ it must hold in $\mathcal{V}$.

THEOREM 22. Let $\mathcal{V}$ be an arithmetical variety such that $\mathcal{V}_{\mathrm{FSI}}$ is a universal class. The following are equivalent:

1. $\mathcal{V}$ has surjective epimorphisms.

2. For all $\mathbf{A}, \mathbf{B} \in \mathcal{V}$ we have that $\mathbf{A} \leq_{e}^{\mathcal{V}} \mathbf{B}$ implies $\mathbf{A}=\mathbf{B}$.

3. For all $\mathbf{A}, \mathbf{B} \in \mathcal{V}_{\mathrm{FSI}}$ we have that $\mathbf{A} \leq_{e}^{\mathcal{V}_{\mathrm{FSI}}} \mathbf{B}$ implies $\mathbf{A}=\mathbf{B}$.

4. $\mathcal{V}_{\mathrm{FSI}}$ has surjective epimorphisms. 
Proof. We prove $(3) \Rightarrow(2)$ which is the only nontrivial implication. Fix $\mathbf{A}, \mathbf{B} \in \mathcal{V}_{\mathrm{FSI}}$ such that $\mathbf{A} \leq \leq_{\mathrm{V}}^{\mathcal{V}_{\mathrm{FSI}}} \mathbf{B}$ and let $b \in B$. We shall see that $b \in A$. By Theorem 3 there is a p.p. $\mathcal{L}$-formula $\varphi(\bar{x}, y)$ defining a function in $\mathcal{V}$, and such that $[\varphi]^{\mathbf{B}}(\bar{a})=b$ for some $\bar{a} \in A^{n}$. Let

$$
\Sigma:=\left\{\varepsilon \mid \varepsilon \text { is a p.p. sentence of } \mathcal{L}_{A} \text { and } \mathbf{B}_{A} \vDash \varepsilon\right\},
$$

and define

$$
\mathcal{K}:=\left\{\mathbf{C} \mid \mathbf{C} \vDash \Sigma \text { and }\left.\mathbf{C}\right|_{\mathcal{L}} \in \mathcal{V}_{\mathrm{FSI}}\right\}
$$

Claim. $\mathcal{K}$ is a universal class.

Since $\mathcal{K}$ is axiomatizable we only need to check that $\mathcal{K}$ is closed under substructures. Let $\mathbf{C} \leq \mathbf{D} \in \mathcal{K}$; clearly $\left.\mathbf{C}\right|_{\mathcal{L}} \in \mathcal{V}_{\text {FSI }}$, so it remains to see that $\mathbf{C} \vDash \Sigma$. As $\mathbf{D} \vDash \Sigma$, Lemma 2 yields a homomorphism $h: \mathbf{B}_{A} \rightarrow \mathbf{E}$ with $\mathbf{E}$ an ultrapower of D. Note that $\mathbf{E} \in \mathcal{K}$. Since $h(\mathbf{A}) \leq_{e}^{\mathcal{V}} h(\mathbf{B})$ and $h(\mathbf{A}), h(\mathbf{B}) \in \mathcal{V}_{\mathrm{FSI}}$, it follows that $h(A)=h(B)$, because there are no proper epic subalgebras in $\mathcal{V}_{\text {FSI }}$. Now $\mathbf{C}$ is an $\mathcal{L}_{A^{-}}$-subalgebra of $\mathbf{D}$, so $h(B)=h(A) \subseteq C$. Finally, since $h(\mathbf{B}) \vDash \Sigma$ and every sentence in $\Sigma$ is existential, we obtain $\mathbf{C} \vDash \Sigma$. This finishes the proof of the claim.

Claim. $\mathbb{V}(\mathcal{K})$ is arithmetical and $\mathbb{V}(\mathcal{K})_{\mathrm{FSI}}=\mathcal{K}$.

To show that $\mathbb{V}(\mathcal{K})$ is arithmetical we can proceed as in the proof of Lemma 21. We prove $\mathbb{V}(\mathcal{K})_{\text {FSI }}=\mathcal{K}$. Note that for $\mathbf{C} \in \mathcal{K}$ we have that $\mathbf{C}$ and $\left.\mathbf{C}\right|_{\mathcal{L}}$ have the same congruences; hence every algebra in $\mathcal{K}$ is FSI. For the other inclusion, Jónsson's lemma [14] produces $\mathbb{V}(\mathcal{K})_{\mathrm{FSI}} \subseteq \mathbb{H S P}_{u}(\mathcal{K})$, and by the first claim $\mathbb{H S P}_{u}(\mathcal{K})=\mathbb{H}(\mathcal{K})$. So, as $\mathbb{H}(\mathcal{K}) \vDash \Sigma$, we have that $\mathbb{V}(\mathcal{K})_{\mathrm{FSI}} \vDash \Sigma$ and thus $\mathbb{V}(\mathcal{K})_{\text {FSI }} \subseteq \mathcal{K}$

Next we want to apply Lemma 21 to $\mathbb{V}(\mathcal{K})$ and $\varphi(\bar{a}, y)$, so we need to check that the hypothesis holds. Take $\mathbf{C} \in \mathcal{K}$ and $\mathbf{S} \leq \mathbf{C}$. Since $\mathcal{K}$ is universal we have $\mathbf{S} \in \mathcal{K}$, and thus $\mathbf{S} \vDash \exists y \varphi(\bar{a}, y)$. Let $t$ be a term such that $\mathbb{V}(\mathcal{K}) \vDash \forall y \varphi(\bar{a}, y) \rightarrow$ $y=t$. Then $b=t^{\mathbf{B}_{A}} \in A$, and we are done.

Every discriminator variety (see [5, Def. 9.3] for the definition) satisfies the hypothesis in Theorem 22. Furthermore, in such a variety every FSI member is simple (i.e., has exactly two congruences). Writing $\mathcal{V}_{\mathrm{S}}$ for the class of simple members in $\mathcal{V}$ we have the following immediate consequence of Theorem 22 .

Corollary 23. For a discriminator variety $\mathcal{V}$ the following are equivalent.

1. $\mathcal{V}$ has surjective epimorphisms.

2. For all $\mathbf{A}, \mathbf{B} \in \mathcal{V}$ we have that $\mathbf{A} \leq_{e}^{\mathcal{V}} \mathbf{B}$ implies $\mathbf{A}=\mathbf{B}$.

3. For all $\mathbf{A}, \mathbf{B} \in \mathcal{V}_{\mathrm{S}}$ we have that $\mathbf{A} \leq_{e} \mathcal{V}_{\mathrm{S}} \mathbf{B}$ implies $\mathbf{A}=\mathbf{B}$.

4. $\mathcal{V}_{\mathrm{S}}$ has surjective epimorphisms.

It is not uncommon for a variety arising as the algebrization of a logic to be a discriminator variety; thus the above corollary could prove helpful in establishing the Beth definability property for such a logic.

Another special case relevant to algebraic logic to which Theorem 22 applies is given by the class of Heyting algebras and its subvarieties (none of these are discriminator varieties with the exception of the class of boolean algebras). Heyting algebras constitute the algebraic counterpart to intuitionistic logic, and 
have proven to be a fertile ground to investigate definability and interpolation properties of intuitionistic logic and its axiomatic extensions by algebraic means (see [1] and its references).

I would like to thank Diego Castaño and Tommaso Moraschini for their insightful discussions during the preparation of this paper. I would also like to thank the anonymous referee for introducing me to the concept of dominions. She/he pointing out that the main theorem of my original submission could be restated as a characterization of dominions (instead of a characterization of epic substructures) provided a significant improvement to the scope of this article.

References

[1] G. Bezhanishvili, T. Moraschini, and J. Raftery, Epimorphisms in varieties of residuated structures, Submitted, 2016.

[2] W. Blok and E. Hoogland, The Beth property in algebraic logic, Studia Logica, vol. 83 (2006), pp. 49-90.

[3] A. Budkin, Dominions in quasivarieties of universal algebras, Studia Logica, vol. 78 (2004), no. 1-2, pp. 107-127.

[4] - Dominions of universal algebras and projective properties, Algebra and Logic, vol. 47 (2008), no. 5, pp. 304-313.

[5] S. Burris and H. SANKA PPANAVAR, A course in universal algebra, Graduate texts in mathematics, Springer-Verlag, 1981.

[6] M. Campercholi and D. Vaggione, Semantical conditions for the definability of functions and relations, Algebra universalis, vol. 76 (2016), no. 1, pp. 71-98.

[7] J. Czelakowski and W. Dziobiak, Congruence distributive quasivarieties whose finitely subdirectly irreducible members form a universal class, algebra universalis, vol. 27 (1990), no. 1, pp. 128-149.

[8] P. Higgins, Epimorphisms and amalgams, Colloquium Mathematicae, vol. 56 (1988), no. 1, pp. 1-17 (eng).

[9] — A short proof of Isbell's zigzag theorem., Pacific J. Math., vol. 144 (1990), no. 1 , pp. $47-50$.

[10] W. Hodges, Model theory, Encyclopedia of Mathematics and its Applications, Cambridge University Press, 1993.

[11] P. Hoffman, A proof of Isbell's zigzag theorem, Journal of the Australian Mathematical Society, vol. 84 (2008), pp. 229-232.

[12] J. Howie, Fundamentals of semigroup theory, LMS monographs, Clarendon Press, 1995.

[13] J. Isbell, Epimorphisms and dominions, Proceedings of the conference on categorical algebra: La jolla 1965, Springer Berlin Heidelberg, 1966, pp. 232-246.

[14] B. Jónsson, Algebras whose congruence lattices are distributive, Mathematica Scandinavica, vol. 21 (1968), no. 1, pp. 110-121.

[15] J. Philip, A proof of Isbell's zig-zag theorem, Journal of Algebra, vol. 32 (1974), no. 2 , pp. $328-331$.

[16] J. Renshaw, On free products of semigroups and a new proof of Isbell's zigzag theorem, Journal of Algebra, vol. 251 (2002), no. 1, pp. 12 - 15.

[17] H. Storrer, An algebraic proof of Isbell's zigzag theorem., Semigroup forum, vol. 12 (1976), pp. 83-88.

[18] D. Vaggione, Infinitary Baker-Pixley Theorem, Submitted, 2016.

[19] D. Wasserman, Epimorphisms and dominions in varieties of lattices, Ph.D. thesis, University of California at Berkeley, 2001. 
CIEM - FACULTAD DE MATEMÁTICA, ASTRONOMíA Y FíSICA (FA.M.A.F.) UNIVERSIDAD NACIONAL DE CÓRDOBA — CIUDAD UNIVERSITARIA CÓRDOBA 5000. ARGENTINA

E-mail: camper@famaf.unc.edu.ar 